\title{
THE VARIANT OF THE GOURSAT PROBLEM FOR THE SIXTH ORDER EQUATION
}

\author{
A. MAHER AND YE. A. UTKKINA
}

\begin{abstract}
Our study is considered as a continuation of results in [1-8]. More precisely we investigate the Goursat problem in class $C^{2+2+2}(D)$ for the equation (1) below by Riemann method variant from the works [8] and [9].
\end{abstract}

\section{Introduction}

In the domain $D=\left\{(x, y, z) ; x_{0}<x<x_{1}, y_{0}<y<y_{1}, z_{0}<z<z_{1}\right\}$ we consider the equation with variable coefficients:

$$
L(u)=\sum_{\alpha, \beta, \gamma=0}^{2} a_{\alpha \beta \gamma}(x, y, z) \frac{\partial^{\alpha+\beta+\gamma} u}{\partial x^{\alpha} \partial y^{\beta} \partial z^{\gamma}}=F(x, y, z) .
$$

It may be considered as a special generalization of the investigation in [1-8]. Many authors studied this problem in the different directions (e.g.,[1-8]). The most general results were obtained in [7]. On the other hand, our aim in this paper is to consider the equation (1) which is a complication to the works [8-11] with higher derivative $u_{x y z}$. This equation plays an important role in approximation and transformation theory and during the description of vibration processes (see, [12]). The problem of integral transformation representation of type of ordinary linear differential operator into others is reduced to the similar equation in [13].

\section{Main Results}

Let $X, Y, Z$ be bounds $D$ for $x=x_{0}, y=y_{0}, z=z_{0}$, respectively. We consider that $a_{222} \equiv 1$, and the smoothness of other coefficients (1) is defined by the inclusions:

$$
a_{\alpha \beta \gamma} \in C^{\alpha+\beta+\gamma}(\bar{D}), \quad F \in C^{2+2+2}(\bar{D}) .
$$

Received April 20, 2005.

2000 Mathematics Subject Classification. 35B, 35J.

Key words and phrases. Sixth order partial differential equation, Goursat problem, Riemann function. 
Here, $C^{\alpha+\beta+\gamma}$ describes the class of continuous in $\bar{D}$ together with derivatives $\partial^{r+s+t}$ $/ \partial x^{r} \partial y^{s} \partial z^{t}(r=0, \ldots, \alpha ; s=0, \ldots, \beta ; t=0, \ldots, \gamma)$.

Problem (Goursat). To find, in class $C^{2+2+2}(\bar{D})$, the solution of equation (1) satisfying the following boundary conditions:

$$
\begin{aligned}
\left.u\right|_{X} & =\varphi(y, z),\left.\quad u\right|_{Y}=\psi(x, z), & \left.u\right|_{Z}=\theta(x, y) ; \\
\left.u_{x}\right|_{X} & =\varphi_{1}(y, z),\left.\quad u_{y}\right|_{Y}=\psi_{1}(x, z), & \left.u_{z}\right|_{Z}=\theta_{1}(x, y) .
\end{aligned}
$$

where

$$
\varphi, \varphi_{1} \in C^{2+2}(\bar{X}) \quad \psi, \psi_{1} \in C^{2+2}(\bar{Y}) \quad \theta, \theta_{1} \in C^{2+2}(\bar{Z}) .
$$

We suppose the satisfaction of the condition in ribs $D$

$$
\varphi\left(y_{0}, z\right)=\psi\left(x_{0}, z\right), \quad \varphi\left(y, z_{0}\right)=\theta\left(x_{0}, y\right), \quad \psi\left(x, z_{0}\right)=\theta\left(x, y_{0}\right) .
$$

Here the agreed values are continuously differentiated. We solve this problem by means of the development of (using) the Riemann method variant from the works [8] and [9].

Riemann function $R(x, y, z, \zeta, \eta, \varsigma)$ is called the solution of following integral equation:

$$
\begin{aligned}
V(x, y, z)- & \int_{\varsigma}^{z}\left[a_{221}(x, y, \gamma) V(x, y, \gamma)-(z-\gamma) a_{220}(x, y, \gamma) V(x, y, \gamma)\right] d \gamma \\
- & \int_{\eta}^{y}\left[a_{212}(x, \beta, z) V(x, \beta, z)-(y-\beta) a_{202}(x, \beta, z) V(x, \beta, z)\right] d \beta \\
- & \int_{\zeta}^{x}\left[a_{122}(\alpha, y, z) V(\alpha, y, z)-(x-\alpha) a_{022}(\alpha, y, z) V(\alpha, y, z)\right] d \alpha \\
+ & \int_{\zeta}^{x} \int_{\eta}^{y}\left[a_{112}(\alpha, \beta, z) V(\alpha, \beta, z)-(y-\beta) a_{102}(\alpha, \beta, z) V(\alpha, \beta, z)\right. \\
& \left.-(x-\alpha) a_{012}(\alpha, \beta, z) V(\alpha, \beta, z)+(x-\alpha)(y-\beta) a_{002}(\alpha, \beta, z) V(\alpha, \beta, z)\right] d \beta d \alpha \\
+ & \int_{\zeta}^{x} \int_{\varsigma}^{z}\left[a_{121}(\alpha, y, \gamma) V(\alpha, y, \gamma)-(z-\gamma) a_{120}(\alpha, y, \gamma) V(\alpha, y, \gamma)\right. \\
& \left.-(x-\alpha) a_{021}(\alpha, y, \gamma) V(\alpha, y, \gamma)+(x-\alpha)(z-\gamma) a_{020}(\alpha,, y, \gamma) V(\alpha, y, \gamma)\right] d \gamma d \alpha \\
+ & \int_{\eta}^{y} \int_{\varsigma}^{z}\left[a_{211}(x, \beta, \gamma) V(x, \beta, \gamma)-(y-\beta) a_{201}(x, \beta, \gamma) V(x, \beta, \gamma)\right. \\
& \left.-(z-\gamma) a_{210}(x, \beta, \gamma) V(x, \beta, \gamma)+(y-\beta)(z-\gamma) a_{200}(x, \beta, \gamma) V(x, \beta, \gamma)\right] d \gamma d \beta \\
- & \int_{\zeta}^{x} \int_{\eta}^{y} \int_{\varsigma}^{z}\left[a_{111}(\alpha, \beta, \gamma) V(\alpha, \beta, \gamma)-(y-\beta) a_{101}(\alpha, \beta, \gamma) V(\alpha, \beta, \gamma)\right. \\
& -(x-\alpha) a_{011}(\alpha, \beta, \gamma) V(\alpha, \beta, \gamma)-(z-\gamma) a_{110}(\alpha, \beta, \gamma) V(\alpha, \beta, \gamma) \\
& +(y-\beta)(z-\gamma) a_{100}(\alpha, \beta, \gamma) V(\alpha, \beta, \gamma)+(x-\alpha)(z-\gamma) a_{010}(\alpha, \beta, \gamma) V(\alpha, \beta, \gamma) \\
& +(x-\alpha)(y-\beta) a_{001}(\alpha, \beta, \gamma) V(\alpha, \beta, \gamma) \\
& \left.-(x-\alpha)(y-\beta)(z-\gamma) a_{000}(\alpha, \beta, \gamma) V(\alpha, \beta, \gamma)\right] d \alpha d \beta d \gamma=1 .
\end{aligned}
$$


which exists and unique (see [14], pp.180). We may verify that the realization of the identity for any function $u$ from the class $C^{2+2+2}(\bar{D})$ directly by the inspection:

$$
\begin{aligned}
(u R)_{x x y y z z}= & R L(u)+\left(u A_{1}\right)_{x x y y z}+\left(u A_{2}\right)_{x x y z z}+\left(u A_{3}\right)_{x y y z z}-\left(u B_{1}\right)_{x y z z} \\
& -\left(u B_{2}\right)_{x x y z}-\left(u B_{3}\right)_{x y y z}-\left(u B_{4}\right)_{x x y y}-\left(u B_{5}\right)_{x x z z}-\left(u B_{6}\right)_{y y z z}+\left(u C_{1}\right)_{x y z} \\
& +\left(u C_{2}\right)_{x z z}+\left(u C_{3}\right)_{x y y}+\left(u C_{4}\right)_{x x y}+\left(u C_{5}\right)_{x x z}+\left(u C_{6}\right)_{y y z}+\left(u C_{7}\right)_{y z z} \\
& -\left(u D_{1}\right)_{x y}-\left(u D_{2}\right)_{x z}-\left(u D_{3}\right)_{y z}-\left(u D_{4}\right)_{z z}-\left(u D_{5}\right)_{y y}-\left(u D_{6}\right)_{x x}+\left(u E_{1}\right)_{x} \\
& +\left(u E_{2}\right)_{y}+\left(u E_{3}\right)_{z}+\left\{u\left(a_{002} R\right)_{z}+u_{x}\left(a_{102} R\right)_{z}+u_{y}\left(a_{012} R\right)_{z}+u_{x y}\left(a_{112} R\right)_{z}\right. \\
& \left.+u_{x x}\left(a_{202} R\right)_{z}+u_{y y}\left(a_{022} R\right)_{z}+u_{x x y}\left(a_{212} R\right)_{z}+u_{x y y}\left(a_{122} R\right)_{z}+u_{x x y y} R_{z}\right\}_{z} \\
& +\left\{u\left(a_{202} R\right)_{x z}+u_{y}\left(a_{212} R\right)_{x z}+u_{y y} R_{x z}\right\}_{x z}+\left\{u\left(a_{022} R\right)_{y z}+u_{x}\left(a_{122} R\right)_{y z}\right. \\
& \left.+u_{x x} R_{y z}\right\}_{y z}+\left\{u R_{x y z}\right\}_{x y z}+\left\{u\left(a_{200} R\right)_{x}+u_{y}\left(a_{210} R\right)_{x}+u_{y y}\left(a_{220} R\right)_{x}\right. \\
& +u_{z}\left(a_{201} R\right)_{x}+u_{z z}\left(a_{202} R\right)_{x}+u_{y z}\left(a_{211} R\right)_{x}+u_{y y z}\left(a_{221} R\right)_{x}+u_{y z z}\left(a_{212} R\right)_{x} \\
& \left.+u_{y y z z} R_{x}\right\}_{x}+\left\{u\left(a_{020} R\right)_{y}+u_{x}\left(a_{120} R\right)_{y}+u_{z}\left(a_{021} R\right)_{y}+u_{x z}\left(a_{121} R\right)_{y}\right. \\
& +u_{x x}\left(a_{220} R\right)_{y}+u_{z z}\left(a_{022} R\right)_{y}+u_{x z z}\left(a_{122} R\right)_{y}+u_{x x z}\left(a_{221} R\right)_{y} \\
& \left.+u_{x x z z} R_{y}\right\}_{y}+\left\{u\left(a_{220} R\right)_{x y}+u_{z}\left(a_{221} R\right)_{x y}+u_{z z} R_{x y}\right\}_{x y},
\end{aligned}
$$

where the notation

$$
\begin{aligned}
& A_{1}=R_{z}-a_{221} R, \quad A_{2}=R_{y}-a_{212} R, \quad A_{3}=R_{x}-a_{122} R \\
& B_{1}=R_{x y}-\left(a_{212} R\right)_{x}-\left(a_{122} R\right)_{y}+a_{112} R, \quad B_{2}=R_{y z}-\left(a_{221} R\right)_{y}-\left(a_{212} R\right)_{z}+a_{211} R, \\
& B_{3}=R_{x z}-\left(a_{221} R\right)_{x}-\left(a_{122} R\right)_{z}+a_{121} R, \quad B_{4}=R_{z z}-\left(a_{221} R\right)_{z}+a_{220} R \\
& \\
& B_{5}=R_{y y}-\left(a_{212} R\right)_{y}+a_{202} R, \quad B_{6}=R_{x x}-\left(a_{122} R\right)_{x}+a_{022} R, \\
& C_{1}=R_{x y z}-\left(a_{221} R\right)_{x y}-\left(a_{212} R\right)_{x z}-\left(a_{122} R\right)_{y z}+\left(a_{112} R\right)_{z} \\
&+\left(a_{211} R\right)_{x}+\left(a_{121} R\right)_{y}-a_{111} R, \\
& C_{2}= R_{x y y}-\left(a_{212} R\right)_{x y}-\left(a_{122} R\right)_{y y}-\left(a_{112} R\right)_{y}+\left(a_{202} R\right)_{x}-a_{102} R \\
& C_{3}= R_{x z z}-\left(a_{221} R\right)_{x z}-\left(a_{122} R\right)_{z z}-\left(a_{121} R\right)_{z}+\left(a_{220} R\right)_{x}-a_{120} R \\
& C_{4}= R_{y z z}-\left(a_{221} R\right)_{y z}-\left(a_{212} R\right)_{z z}+\left(a_{211} R\right)_{z}+\left(a_{220} R\right)_{y}-a_{210} R \\
& C_{5}= R_{y y z}-\left(a_{221} R\right)_{y y}-\left(a_{212} R\right)_{y z}+\left(a_{211} R\right)_{y}+\left(a_{202} R\right)_{z}-a_{201} R, \\
& C_{6}= R_{x x z}-\left(a_{221} R\right)_{x x}-\left(a_{122} R\right)_{x z}+\left(a_{121} R\right)_{x}+\left(a_{022} R\right)_{y}-a_{021} R \\
& C_{7}= R_{x x y}-\left(a_{212} R\right)_{x x}-\left(a_{122} R\right)_{x y}+\left(a_{112} R\right)_{x}+\left(a_{022} R\right)_{y}-a_{012} R \\
& D_{1}= R_{x y z z}-\left(a_{221} R\right)_{x y z}-\left(a_{212} R\right)_{x z z}-\left(a_{122} R\right)_{y z z}+\left(a_{112} R\right)_{z z}+\left(a_{211} R\right)_{x z} \\
&+\left(a_{121} R\right)_{y z}+\left(a_{220} R\right)_{x y}-\left(a_{111} R\right)_{z}-\left(a_{120} R\right)_{y}-\left(a_{210} R\right)_{x}+a_{110} R \\
& D_{2}= R_{x y y z}-\left(a_{221} R\right)_{x y y}-\left(a_{212} R\right)_{x y z}-\left(a_{122} R\right)_{y y z}+\left(a_{112} R\right)_{y z}+\left(a_{211} R\right)_{x y} \\
&+\left(a_{121} R\right)_{y y}+\left(a_{202} R\right)_{x z}-\left(a_{111} R\right)_{y}-\left(a_{102} R\right)_{z}-\left(a_{201} R\right)_{x}+a_{101} R
\end{aligned}
$$




$$
\begin{aligned}
D_{3}= & R_{x x y z}-\left(a_{221} R\right)_{x x y}-\left(a_{212} R\right)_{x x z}-\left(a_{122} R\right)_{x y z}+\left(a_{112} R\right)_{x z}+\left(a_{211} R\right)_{x x} \\
& +\left(a_{121} R\right)_{x y}+\left(a_{022} R\right)_{y z}-\left(a_{111} R\right)_{x}-\left(a_{021} R\right)_{y}-\left(a_{012} R\right)_{z}+a_{011} R, \\
D_{4}= & R_{x x y y}-\left(a_{212} R\right)_{x x y}-\left(a_{122} R\right)_{x y y}+\left(a_{112} R\right)_{x y}+\left(a_{202} R\right)_{x x} \\
& +\left(a_{022} R\right)_{y y}-\left(a_{102} R\right)_{x}-\left(a_{012} R\right)_{y}+a_{002} R \\
D_{5}= & R_{x x z z}-\left(a_{221} R\right)_{x x z}-\left(a_{122} R\right)_{x z z}+\left(a_{121} R\right)_{x z}+\left(a_{220} R\right)_{x x} \\
& +\left(a_{022} R\right)_{z z}-\left(a_{120} R\right)_{x}-\left(a_{012} R\right)_{z}+a_{020} R \\
D_{6}= & R_{y y z z}-\left(a_{221} R\right)_{y y z}-\left(a_{212} R\right)_{y z z}+\left(a_{211} R\right)_{y z}+\left(a_{220} R\right)_{y y} \\
& +\left(a_{202} R\right)_{z z}-\left(a_{210} R\right)_{y}-\left(a_{201} R\right)_{z}+a_{200} R \\
E_{1}= & R_{x y y z z}-\left(a_{221} R\right)_{x y y z}-\left(a_{212} R\right)_{x y z z}-\left(a_{122} R\right)_{y y z z}+\left(a_{112} R\right)_{y z z}+\left(a_{211} R\right)_{x y z} \\
& +\left(a_{121} R\right)_{y y z}+\left(a_{220} R\right)_{x y y}+\left(a_{202} R\right)_{x z z}-\left(a_{111} R\right)_{y z}-\left(a_{102} R\right)_{z z}-\left(a_{120} R\right)_{y y} \\
& -\left(a_{210} R\right)_{x y}-\left(a_{201} R\right)_{x z}+\left(a_{110} R\right)_{y}+\left(a_{101} R\right)_{z}+\left(a_{200} R\right)_{x}-a_{100} R, \\
E_{2}= & R_{x x y z z}-\left(a_{221} R\right)_{x x y z}-\left(a_{212} R\right)_{x x z z}-\left(a_{122} R\right)_{x y z z}+\left(a_{112} R\right)_{x z z}+\left(a_{211} R\right)_{x x z} \\
& +\left(a_{121} R\right)_{x y z}+\left(a_{220} R\right)_{x x y}+\left(a_{022} R\right)_{y z z}-\left(a_{111} R\right)_{x z}-\left(a_{120} R\right)_{x y}-\left(a_{210} R\right)_{x x} \\
& -\left(a_{021} R\right)_{y z}-\left(a_{012} R\right)_{z z}+\left(a_{110} R\right)_{x}+\left(a_{011} R\right)_{z}+\left(a_{020} R\right)_{y}-a_{010} R, \\
E_{3}= & R_{x x y y z}-\left(a_{221} R\right)_{x x y y}-\left(a_{212} R\right)_{x x y z}-\left(a_{122} R\right)_{x y y z}+\left(a_{112} R\right)_{x y z}+\left(a_{211} R\right)_{x x y} \\
& +\left(a_{121} R\right)_{x y y}+\left(a_{202} R\right)_{x x z}+\left(a_{022} R\right)_{y y z}-\left(a_{111} R\right)_{x y}-\left(a_{102} R\right)_{x z}-\left(a_{201} R\right)_{x x} \\
& -\left(a_{021} R\right)_{y y}-\left(a_{012} R\right)_{y z}+\left(a_{101} R\right)_{x}+\left(a_{011} R\right)_{y}+\left(a_{002} R\right)_{z}-a_{001} R .
\end{aligned}
$$

Here $a_{\alpha \beta \gamma}$ depend on $(x, y, z)$, while $R$ and its derivatives depend on $(x, y, z, \zeta, \eta, \varsigma)$. The derivation (6) is a heuristics problem. The easiest way to check the identity itself is the direct calculation. Here we consider that $R$ with its first there arguments satisfies the adjoint equation (1)

$$
\begin{aligned}
L^{*}(V) \equiv & V_{x x y y z z}-\left(a_{221} V\right)_{x x y y z}-\left(a_{211} V\right)_{x x y z z}-\left(a_{121} V\right)_{x y y z z}+\left(a_{112} V\right)_{x y z z} \\
& +\left(a_{211} V\right)_{x x y z}+\left(a_{121} V\right)_{x y y z}+\left(a_{220} V\right)_{x x y y}+\left(a_{202} V\right)_{x x z z}+\left(a_{022} V\right)_{y y z z} \\
& -\left(a_{110} V\right)_{x y z}-\left(a_{102} V\right)_{x z z}-\left(a_{120} V\right)_{x y y}-\left(a_{210} V\right)_{x x y}-\left(a_{201} V\right)_{x x z} \\
& -\left(a_{021} V\right)_{y y z}+\left(a_{012} V\right)_{y z z}+\left(a_{110} V\right)_{x y}+\left(a_{101} V\right)_{x z}+\left(a_{011} V\right)_{y z}+\left(a_{002} V\right)_{z z} \\
& +\left(a_{020} V\right)_{y y}+\left(a_{200} V\right)_{x x}-\left(a_{100} V\right)_{x}-\left(a_{010} V\right)_{y}-\left(a_{001} V\right)_{z}-a_{000} V=0 .
\end{aligned}
$$

It directly follows from (5)that

$$
\begin{aligned}
& A_{1}(x, y, z, x, y, z) \equiv A_{2}(x, y, z, x, y, z) \equiv A_{3}(x, y, z, x, y, z) \\
\equiv & B_{1}(x, y, z, x, y, z) \equiv B_{2}(x, y, z, x, y, z) \equiv B_{3}(x, y, z, x, y, z) \\
\equiv & B_{4}(x, y, z, x, y, z) \equiv B_{5}(x, y, z, x, y, z) \equiv C_{1}(x, y, z, x, y, z) \\
\equiv & C_{2}(x, y, z, x, y, z) \equiv C_{3}(x, y, z, x, y, z) \equiv C_{4}(x, y, z, x, y, z) \\
\equiv & C_{5}(x, y, z, x, y, z) \equiv C_{6}(x, y, z, x, y, z) \equiv C_{7}(x, y, z, x, y, z) \\
\equiv & D_{1}(x, y, z, x, y, z) \equiv D_{2}(x, y, z, x, y, z) \equiv D_{3}(x, y, z, x, y, z) \\
\equiv & D_{4}(x, y, z, x, y, z) \equiv D_{5}(x, y, z, x, y, z) \equiv D_{6}(x, y, z, x, y, z) \\
\equiv & E_{1}(x, y, z, x, y, z) \equiv E_{2}(x, y, z, x, y, z) \equiv E_{3}(x, y, z, x, y, z) \equiv 0 .
\end{aligned}
$$


Now, we put for (6) $x=\alpha, y=\beta, z=\gamma, \zeta=x, \eta=y$. and $\varsigma=z$, and calculate the triple integral in the bounds $x_{0}<\alpha<x, y_{0}<\beta<y, z_{0}<\gamma<z$ from the right and left parts. Here, taking into account the identity (9), boundary conditions (3) and the fact that $u(x, y, z)$ is the solution of the equation (1) we obtain the formula:

$$
\begin{aligned}
& u_{x y z}=\int_{x_{0}}^{x} \int_{y_{0}}^{y} \int_{z_{0}}^{z} R(\alpha, \beta, \gamma, x, y, z) d \gamma d \beta d \alpha+\varphi_{1 y z}(y, z) R\left(x_{0}, y, z, x, y, z\right) \\
& +\psi_{1 x z}(x, z) R\left(x, y_{0}, z, x, y, z\right)-\varphi_{1 y z}\left(y_{0}, z\right) R\left(x_{0}, y_{0}, z, x, y, z\right) \\
& +\theta_{1 x y}(x, y) R\left(x, y, z_{0}, x, y, z\right)-\varphi_{1 y z}\left(y, z_{0}\right) R\left(x_{0}, y, z_{0}, x, y, z\right) \\
& -\psi_{1 x z}\left(x, z_{0}\right) R\left(x, y_{0}, z_{0}, x, y, z\right)+\varphi_{1 y z}\left(y_{0}, z_{0}\right) R\left(x_{0}, y_{0}, z_{0}, x, y, z\right) \\
& -A_{1}\left(x_{0}, y, z\right) \varphi_{1 y}(y, z)-A_{1}\left(x, y_{0}, z\right) \psi_{1 y}(x, z)+A_{1}\left(x_{0}, y_{0}, z\right) \varphi_{1 y}\left(y_{0}, z\right) \\
& -A_{1}\left(x, y, z_{0}\right) \theta_{x y}(x, y)+A_{1}\left(x_{0}, y, z_{0}\right) \varphi_{1 y}\left(y, z_{0}\right)+A_{1}\left(x, y_{0}, z_{0}\right) \psi_{1 x}\left(x, z_{0}\right) \\
& -A_{1}\left(x_{0}, y_{0}, z_{0}\right) \varphi_{1 y}\left(y_{0}, z_{0}\right)-A_{2}\left(x_{0}, y, z\right) \varphi_{1 z}(y, z)-A_{2}\left(x, y_{0}, z\right) \psi_{x y}(x, z) \\
& +A_{2}\left(x_{0}, y_{0}, z\right) \varphi_{1 z}\left(y_{0}, z\right)-A_{2}\left(x, y, z_{0}\right) \theta_{1 x}(x, y)+A_{2}\left(x_{0}, y, z_{0}\right) \varphi_{1 y}\left(y, z_{0}\right) \\
& +A_{2}\left(x, y_{0}, z_{0}\right) \theta_{1 x}\left(x, y_{0}\right)-A_{2}\left(x_{0}, y_{0}, z_{0}\right) \varphi_{1 z}\left(y_{0}, z_{0}\right)-A_{3}\left(x_{0}, y, z\right) \varphi_{y z}(y, z) \\
& -A_{3}\left(x, y_{0}, z\right) \psi_{1 z}(x, z)+A_{3}\left(x_{0}, y_{0}, z\right) \psi_{1 z}\left(x_{0}, z\right)-A_{3}\left(x, y, z_{0}\right) \theta_{1 y}(x, y) \\
& +A_{3}\left(x_{0}, y, z_{0}\right) \theta_{1 y}\left(x_{0}, y\right)+A_{3}\left(x, y_{0}, z_{0}\right) \psi_{1 z}\left(x, z_{0}\right)-A_{3}\left(x_{0}, y_{0}, z_{0}\right) \psi_{1 z}\left(x_{0}, z_{0}\right) \\
& +B_{1}\left(x_{0}, y, z\right) \varphi_{z}(y, z)+B_{1}\left(x, y_{0}, z\right) \psi_{z}(x, z)-B_{1}\left(x_{0}, y_{0}, z\right) \varphi_{z}\left(y_{0}, z\right) \\
& +B_{1}\left(x, y, z_{0}\right) \theta_{1}(x, y)-B_{1}\left(x_{0}, y, z_{0}\right) \theta_{1}\left(x_{0}, y\right)-B_{1}\left(x, y_{0}, z_{0}\right) \theta_{1}\left(x, y_{0}\right) \\
& +B_{1}\left(x_{0}, y_{0}, z_{0}\right) \theta_{1}\left(x_{0}, y_{0}\right)+B_{2}\left(x_{0}, y, z\right) \varphi_{1}(y, z)+B_{2}\left(x, y_{0}, z_{0}\right) \psi_{1 x}(x, z) \\
& -B_{2}\left(x_{0}, y_{0}, z\right) \varphi_{1}\left(y_{0}, z\right)+B_{2}\left(x, y, z_{0}\right) \theta(x, y)-B_{2}\left(x_{0}, y, z_{0}\right) \varphi_{1}\left(y, z_{0}\right) \\
& -B_{2}\left(x, y_{0}, z_{0}\right) \theta_{x}\left(x, y_{0}\right)+B_{2}\left(x_{0}, y_{0}, z_{0}\right) \varphi_{1}\left(y_{0}, z_{0}\right)+B_{3}\left(x_{0}, y, z\right) \varphi_{y}(y, z) \\
& +B_{3}\left(x, y_{0}, z\right) \psi_{1}(x, z)-B_{3}\left(x_{0}, y_{0}, z\right) \psi_{1}\left(x_{0}, z\right)+B_{3}\left(x, y, z_{0}\right) \theta_{y}(x, y) \\
& -B_{3}\left(x_{0}, y, z_{0}\right) \varphi_{y}\left(y, z_{0}\right)-B_{3}\left(x, y_{0}, z_{0}\right) \psi_{1}\left(x, z_{0}\right)+B_{3}\left(x_{0}, y_{0}, z_{0}\right) \psi_{1}\left(x_{0}, z_{0}\right) \\
& -C_{1}\left(x_{0}, y, z\right) \varphi(y, z)-C_{1}\left(x, y_{0}, z\right) \psi(x, z)+C_{1}\left(x_{0}, y_{0}, z\right) \varphi\left(y_{0}, z\right) \\
& -C_{1}\left(x, y, z_{0}\right) \theta(x, y)+C_{1}\left(x_{0}, y, z_{0}\right) \varphi\left(y, z_{0}\right)+C_{1}\left(x, y_{0}, z_{0}\right) \psi\left(x, z_{0}\right) \\
& -C_{1}\left(x_{0}, y_{0}, z_{0}\right) \varphi\left(y_{0}, z_{0}\right) \\
& +\int_{x_{0}}^{x}\left\{B_{6}\left(\alpha, y_{0}, z\right) \psi_{1 z}(\alpha, z)+B_{6}\left(\alpha, y, z_{0}\right) \theta_{1 y}(\alpha, y)+B_{6}\left(\alpha, y_{0}, z_{0}\right) \psi_{1 z}\left(\alpha, z_{0}\right)\right. \\
& -C_{6}\left(\alpha, y_{0}, z\right) \psi_{1}(\alpha, z)-C_{6}\left(\alpha, y, z_{0}\right) \theta_{y}(\alpha, y)+C_{6}\left(\alpha, y_{0}, z_{0}\right) \psi_{1}\left(\alpha, z_{0}\right) \\
& -C_{7}\left(\alpha, y_{0}, z\right) \psi_{z}(\alpha, z)-C_{7}\left(\alpha, y, z_{0}\right) \theta_{1}(\alpha, y)+C_{7}\left(\alpha, y_{0}, z_{0}\right) \theta_{1}\left(\alpha, y_{0}\right) \\
& \left.+D_{3}\left(\alpha, y_{0}, z\right) \psi(\alpha, z)+D_{3}\left(\alpha, y, z_{0}\right) \theta(\alpha, y)-D_{3}\left(\alpha, y_{0}, z_{0}\right) \psi\left(\alpha, z_{0}\right)\right\} d \alpha
\end{aligned}
$$




$$
\begin{aligned}
+ & \int_{y_{0}}^{y}\left\{B_{5}\left(x, \beta, z_{0}\right) \theta_{1 x}(x, \beta)+B_{5}\left(x_{0}, \beta, z\right) \varphi_{1 z}(\beta, z)-B_{5}\left(x_{0}, \beta, z_{0}\right) \varphi_{1 z}\left(\beta, z_{0}\right)\right. \\
& -C_{2}\left(x_{0}, \beta, z\right) \varphi_{z}(\beta, z)-C_{2}\left(x, \beta, z_{0}\right) \theta_{1}(x, \beta)+C_{2}\left(x_{0}, \beta, z_{0}\right) \theta_{1}\left(x_{0}, \beta\right) \\
& -C_{5}\left(x_{0}, \beta, z\right) \varphi_{1}(\beta, z)-C_{5}\left(x, \beta, z_{0}\right) \theta_{x}(x, \beta)+C_{5}\left(x_{0}, \beta, z_{0}\right) \varphi_{1}\left(\beta, z_{0}\right) \\
& \left.+D_{2}\left(x_{0}, \beta, z\right) \varphi(\beta, z)+D_{2}\left(x, \beta, z_{0}\right) \theta(x, \beta)-D_{2}\left(x_{0}, \beta, z_{0}\right) \varphi\left(\beta, z_{0}\right)\right\} d \beta \\
+ & \int_{z_{0}}^{z}\left\{B_{4}\left(x_{0}, y, \gamma\right) \varphi_{1 y}(y, \gamma)+B_{4}\left(x, y_{0}, \gamma\right) \psi_{1 x}(x, \gamma)-B_{4}\left(x_{0}, y_{0}, \gamma\right) \varphi_{1 y}\left(y_{0}, \gamma\right)\right. \\
& -C_{3}\left(x_{0}, y, \gamma\right) \varphi_{y}(y, \gamma)-C_{3}\left(x, y_{0}, \gamma\right) \psi_{1}(x, \gamma)+C_{3}\left(x_{0}, y_{0}, \gamma\right) \psi_{1}\left(x_{0}, \gamma\right) \\
& -C_{4}\left(x_{0}, y, \gamma\right) \varphi_{1}(y, \gamma)-C_{4}\left(x, y_{0}, \gamma\right) \psi_{x}(x, \gamma)+C_{4}\left(x_{0}, y_{0}, \gamma\right) \varphi_{1}\left(y_{0}, \gamma\right) \\
& \left.+D_{1}\left(x_{0}, y, \gamma\right) \varphi(y, \gamma)+D_{1}\left(x, y_{0}, \gamma\right) \psi(x, \gamma)-D_{1}\left(x_{0}, y_{0}, \gamma\right) \varphi\left(y_{0}, \gamma\right)\right\} d \gamma \\
+ & \int_{x_{0}}^{x} \int_{y_{0}}^{y}\left\{D_{4}\left(\alpha, \beta, z_{0}\right) \theta_{1}(\alpha, \beta)-E_{1}\left(\alpha, \beta, z_{0}\right) \theta(\alpha, \beta)\right\} d \beta d \alpha \\
+ & \int_{x_{0}}^{x} \int_{z_{0}}^{z}\left\{D_{5}\left(\alpha, y_{0}, \gamma\right) \psi_{1}(\alpha, \gamma)-E_{2}\left(\alpha,, y_{0}, \gamma\right) \psi(\alpha, \gamma)\right\} d \gamma d \alpha \\
+ & \int_{y_{0}}^{y} \int_{z_{0}}^{z}\left\{D_{6}\left(x_{0}, \beta, \gamma\right) \varphi_{1}(\beta, \gamma)-E_{1}\left(x_{0}, \beta, \gamma\right) \varphi(\beta, \gamma)\right\} d \gamma d \beta .
\end{aligned}
$$

Hence, marking the right part (10) without the first addend as $H(x, y, z, x, y, z)$, we come to the solution of the problem under our consideration (1) and (3):

$$
\begin{aligned}
u(x, y, z)= & \varphi(y, z)+\psi(x, z)+\theta(x, y)-\varphi\left(y_{0}, z\right)-\psi\left(x, z_{0}\right)-\theta\left(x_{0}, y\right)+\varphi\left(y_{0}, z_{0}\right) \\
& +\int_{x_{0}}^{x} \int_{y_{0}}^{y} \int_{z_{0}}^{z} H(\alpha, \beta, \gamma, \alpha, \beta, \gamma) d \gamma d \beta d \alpha \\
& +\int_{x_{0}}^{x} \int_{x_{0}}^{\alpha} \int_{y_{0}}^{y} \int_{y_{0}}^{\beta} \int_{z_{0}}^{z} \int_{z_{0}}^{\gamma} R\left(t_{1}, t_{2}, t_{3}, \alpha, \beta, \gamma\right) F\left(t_{1}, t_{2}, t_{3}\right) d t_{3} d \gamma d t_{2} d \beta d t_{1} d \alpha .
\end{aligned}
$$

Smoothness (differentiable) conditions (2) for coefficients of the equation (1) provide belonging to the solution class $C^{2+2+2}(D)$. If we consider the functions $\varphi, \varphi_{1}, \psi, \psi_{1}, \theta, \theta_{1}$ as derivatives we many consider (10) and (11) as a general representation of the solution for the equation (1), in the similar way as it is done in [15] for the equation with the higher derivative $u_{x y}$. Therefore, our results may be formulated as follows:

Theorem. The Goursat problem for the equation (1) with the boundary conditions (3) and conditions (4) has the solution which is written by means of the formula (11).

\section{Special cases}

Formulas (10) and (11) provide for the solution of the problem (1)under the conditions (3) in quadratures if the explicit form $R$ is known. We shall mention some of these cases that were obtained by means of direct solution (5).

Let us consider seven coefficients of the equation (1) $a_{221}, a_{212}, a_{122}, a_{112}, a_{211}, a_{121}$, $a_{111}$ as different from zero while all the others are identically equal to zero. Here (5) has 
the same form as the equation (4) from the paper [11]. We consider that the following identities are satisfied:

$$
\begin{aligned}
& \frac{\partial a_{220}}{\partial y}+a_{220} a_{121}-a_{120} \equiv 0, \quad \frac{\partial a_{220}}{\partial y}+a_{220} a_{211}-a_{210} \equiv 0, \\
& \frac{\partial a_{121}}{\partial y}+a_{121} a_{211}-a_{111} \equiv 0, \quad \frac{\partial a_{120}}{\partial y}+a_{120} a_{211}-a_{110} \equiv 0 .
\end{aligned}
$$

Then, it follows from [11] that the Riemann function has the form:

$$
R(x, y, z, \zeta, \eta, \varsigma)=\exp \left[\int_{\varsigma}^{z} a_{220}(\zeta, \eta, \gamma) d \gamma+\int_{\zeta}^{x} a_{121}(\alpha, \beta, z) d \alpha+\int_{\eta}^{y} a_{211}(\alpha, \beta, z) d \beta\right] .
$$

If the last identity (12) is non-zero but of the product $\lambda(x) \mu(y) \nu(z)$ and when

$$
a_{220}=A(z)+\delta x y, \quad a_{121}=B(x)+\delta y z, \quad a_{211}=C(y)+\delta x z,
$$

by using the formulas (18) and (25) from [11] we obtain the following

$$
R(x, y, z, \zeta, \eta, \varsigma)=R_{0}(x, y, z, \zeta, \eta, \varsigma)_{0} F_{2}(1,1, \omega),
$$

where ${ }_{0} F_{2}$ - is the generalized (distributed) hyper-geometrical function (see, [16] pp,20) while

$$
\begin{aligned}
R_{0} & \equiv \exp \left[\int_{\zeta}^{x} B(\alpha) d \alpha+\int_{\eta}^{y} C(\beta) d \beta+\int_{\varsigma}^{z} A(\gamma) d \gamma+\delta(x y z-\zeta \eta \varsigma)\right], \\
\omega & =\int_{\zeta}^{x} \lambda(\alpha) d \alpha \int_{\beta}^{y} \mu(\beta) d \beta \int_{\varsigma}^{z} \nu(\gamma) d \gamma
\end{aligned}
$$

In the similar manner we can also calculate $V$ in other cases when in (5) only one of coefficients $a_{\alpha \beta \gamma}$, having a defined structure, is different form the identical zero.

\section{References}

[1] D. Colton, Pseudoparabolic equations in one space variable, Differential Equations 12(1972), 559-565.

[2] W. Rundell and M. Stecher, Remarks concerning of solutions of peseudoparabolic equation, Proc. Amer. Math. Soc. 63 (1977), 77-81.

[3] W. Rundell, The costruction of solutions to pesudoparabolic equation on noncylindrical domains, Differential Equations 27 (1978), 394-404.

[4] W. Rundell and M. Stecher, The uniqueness class for the Cauchy problem for pesudoparabolic equation, Proc. Amer. Math. Soc. 76 (1979), 253-257.

[5] V. A. Vodakhova, The boundary problem with the A. M. Nakhushev nonlocal condition for a peseudoparabolic equation, Differential Equations 18(1982), 280-285.

[6] M. Kh. Shkhanukov, On the method of solving boundary problems for third order equations, Report of the Russian Academy of Sciences (RRAS) 265(1982), 1327-1330. 
[7] A. P. Soldatov and M. Kh. Shkhanukov, Boundary problems with the A. A. Samarskiy general nonlocal condition for peseudoparabolic equation of higher order, Report of the Russian Academy of Sciences (RRAS) 297(1987), 547-552.

[8] V. I. Zhegalov and Ye. A. Utkkina, On the pseudoparabolic equation of the third order, Izvestia, VUZov: Matematka 10 (1999), 73-76.

[9] V. I. Zhegalov, The three-dimensional analog of the Goursat problem" (Non-classical and mixed-type equations, Novosibirsk), (1999), 73-76.[in Russian]

[10] M. K. Fage, The Cauchy problem for the Bianky equation, Mathematical Collection 45 (1958), 281-322.

[11] V. I. Zhegalov, On the three-dimenisonal Rimann function, Siberian Mathematical Journal 36(1997), 1074-1079.

[12] B. A. Bondarenko, The basic system of polynomial and quasi-polynomial solutions for the partial equations, Tashkent: FAN (1987), p.146.

[13] M. K. Fage, Operational and analytical functions of an independent variable, Work of Moscow Math. Society 7(1958), 227-268.[in Russian]

[14] G. Muenz, Integral equations, Leningrad, Moscow, State Technical and Theoretical Prress 1 (1934), p.330.[in Russian]

[15] A. V. Bitsadze, Same classes of partial differential equations, Moscow, Nauka (1981), p.448.[in Russian]

[16] J. Kampe de Ferier, R. Campbell, G. Petio and T. Voegel, Functions of mathematical physics, Moscow, State Press for Physics and Mathematics (1963), p.102.[in Russian]

P.O. Box 2064, Department of Mathematics, Makkah, The Teacher's of College in Makkah, Kingdam of Saudi Arabia (K.S.A).

E-mail: a_maher69@yahoo.com

Department of Differential Equations, Kazan State University, 18 Kremlyovskaya St., Kazan, 420008, Russia. 